\title{
Spontaneous breaking of Weyl quadratic gravity to Einstein action and Higgs potential
}

\author{
D.M. Ghilencea \\ Department of Theoretical Physics, National Institute of Physics and Nuclear Engineering, \\ Bucharest 07r125, Romania \\ E-mail: dumitru.ghilencea@cern.ch
}

AbstRACT: We consider the (gauged) Weyl gravity action, quadratic in the scalar curvature $(\tilde{R})$ and in the Weyl tensor $\left(\tilde{C}_{\mu \nu \rho \sigma}\right)$ of the Weyl conformal geometry. In the absence of matter fields, this action has spontaneous breaking in which the Weyl gauge field $\omega_{\mu}$ becomes massive (mass $m_{\omega} \sim$ Planck scale) after "eating" the dilaton in the $\tilde{R}^{2}$ term, in a Stueckelberg mechanism. As a result, one recovers the Einstein-Hilbert action with a positive cosmological constant and the Proca action for the massive Weyl gauge field $\omega_{\mu}$. Below $m_{\omega}$ this field decouples and Weyl geometry becomes Riemannian. The EinsteinHilbert action is then just a "low-energy" limit of Weyl quadratic gravity which thus avoids its previous, long-held criticisms. In the presence of matter scalar field $\phi_{1}$ (Higgs-like), with couplings allowed by Weyl gauge symmetry, after its spontaneous breaking one obtains in addition, at low scales, a Higgs potential with spontaneous electroweak symmetry breaking. This is induced by the non-minimal coupling $\xi_{1} \phi_{1}^{2} \tilde{R}$ to Weyl geometry, with Higgs mass $\propto \xi_{1} / \xi_{0}$ ( $\xi_{0}$ is the coefficient of the $\tilde{R}^{2}$ term). In realistic models $\xi_{1}$ must be classically tuned $\xi_{1} \ll \xi_{0}$. We comment on the quantum stability of this value.

Keywords: Classical Theories of Gravity, Effective Field Theories, Higgs Physics, Spontaneous Symmetry Breaking

ARXIV EPRINT: 1812.08613 


\section{Contents}

1 Motivation 1

2 Spontaneous breaking of Weyl gauge symmetry 2

2.1 From Weyl conformal geometry to a Riemannian description 2

2.2 Spontaneous breaking from Weyl gauge symmetry to Einstein gravity 3

3 Adding matter fields $\quad 7$

$\begin{array}{lll}3.1 & \text { Spontaneous breaking of Weyl gauge symmetry } & 7\end{array}$

$\begin{array}{ll}3.2 \text { Electroweak symmetry breaking } & 10\end{array}$

$\begin{array}{lll}4 & \text { Conclusions } & 10\end{array}$

\section{Motivation}

Introduced a century ago, Weyl's conformal geometry [1-5] is a scalar-vector-tensor theory of gravity that is a generalization of Brans-Dicke-Jordan theory of gravity [6-8]. The latter can be recovered in the limit of vanishing Weyl gauge field. Weyl's conformal geometry was applied to model building beyond SM long ago [9, 10], with recent renewed interest [11-22].

In the absence of matter and up to topological terms [22], the Weyl gravity action is the sum of two quadratic terms: the square of the Weyl-geometry scalar curvature term, $\tilde{R}^{2}$, and the square of the Weyl-tensor: $\tilde{C}^{2} \equiv \tilde{C}_{\mu \nu \rho \sigma} \tilde{C}^{\mu \nu \rho \sigma}$. These generalise their counterparts $R^{2}$ and $C^{2}$ of the Riemannian geometry, to include effects due to the Weyl gauge field

$\omega_{\mu}$. Indeed, in Weyl geometry $\nabla_{\mu} g_{\nu \sigma} \propto \omega_{\mu} g_{\rho \sigma}$, so the Weyl connection coefficients $\tilde{\Gamma}_{\mu \nu}^{\rho}$ are not determined by the metric alone (as in the Riemannian case), but also depend on $\omega_{\mu}$. Then $\tilde{R}^{2}$ (or $\tilde{C}^{2}$ ) can be expressed in terms of their Riemannian counterparts $R^{2}\left(C^{2}\right)$ plus a function of $\omega_{\mu}$, and become equal if $\omega_{\mu}=0$ (decoupled); in this limit, Weyl geometry becomes Riemannian. We consider only a torsion-free Weyl geometry.

In section 2 we show that in the absence of matter fields, Weyl quadratic gravity has spontaneous breaking via a Stueckelberg mechanism [23] in which the Weyl gauge field $\omega_{\mu}$ becomes massive after "eating" the Goldstone (dilaton) field $\left(\phi_{0}\right) ; \phi_{0}$ is "extracted" from the $\tilde{R}^{2}$ term which propagates an extra scalar field. Below the mass $m_{\omega}$ of the Weyl gauge field, $m_{\omega} \sim$ Planck scale, this field decouples, Weyl geometry becomes Riemannian and we recover the Einstein-Hilbert action and a positive cosmological constant. So EinsteinHilbert action is a "low-energy" limit of Weyl quadratic gravity which thus avoids previous long-held criticisms against it, see e.g. [2-4]. No additional matter field (scalar, etc.) is required.

The Stueckelberg mechanism of breaking is known in local Weyl-invariant models but these contain, however, additional (matter) scalars and are linear (rather than quadratic) 
in the scalar curvature $\tilde{R}$ of Weyl geometry $[9,10]$ (see also recent $[15,16]$ ). In these models the Einstein action follows from the presence of a scalar matter field $\phi$ with coupling $\phi^{2} \tilde{R}$, absent in our quadratic Weyl action without matter. To our knowledge, the breaking we study in the "pure" geometric case of $\tilde{R}^{2}$ and $\tilde{C}^{2}$-terms only, was not yet discussed. Our goal is to show that this symmetry breaking mechanism is still at work in this case.

In section 3 we also consider the presence of matter fields in addition to Weyl quadratic action. We study the case of the SM Higgs field $\left(\phi_{1}\right)$ with all dimension-four couplings allowed by Weyl gauge symmetry. We show that the Stueckelberg mechanism is still present, with an additional benefit: the Higgs potential has spontaneous electroweak symmetry breaking. This follows from a Weyl-invariant non-minimal coupling $\xi_{1} \phi_{1}^{2} \tilde{R}$; the higgs mass becomes $\propto \xi_{1} / \xi_{0}$ (Planck units), where $\xi_{0}$ is the coefficient of the $\tilde{R}^{2}$ term.

There is also an ultraviolet (UV) motivation to study Weyl gauge symmetry: this can play a role in early cosmology when effective field theory at short distances becomes nearly conformal. Then models with Weyl (gauged) symmetry [9-22], conformal or global scale symmetry [24-45] provide an interesting setup for UV model building. It is possible that Weyl quadratic gravity be a renormalizable theory, similar to (Riemannian) $R^{2}$ gravity [46] Thus Weyl gauge symmetry is interesting for studying the SM in the presence of gravity, with all scales generated spontaneously. This symmetry may even be respected at the quantum level $[28-36,47]$. This opens the possibility to solve the hierarchy problem by endowing the SM with spontaneously broken Weyl gauge symmetry [13-15, 22].

\section{Spontaneous breaking of Weyl gauge symmetry}

\subsection{From Weyl conformal geometry to a Riemannian description}

Let us review some aspects of Weyl geometry needed when discussing Weyl gauge invariance of the action. A (local) conformal transformation of the metric and of a field $\phi$ is given by ${ }^{1}$

$$
\begin{aligned}
g_{\mu \nu} & \rightarrow g_{\mu \nu}^{\prime}=e^{2 \alpha(x)} g_{\mu \nu}, \\
\phi & \rightarrow \phi^{\prime}=e^{-\alpha(x) \Delta} \phi .
\end{aligned}
$$

Then $g^{\mu \nu \prime}=e^{-2 \alpha(x)} g^{\mu \nu}$ and $\sqrt{g^{\prime}}=e^{4 \alpha(x)} \sqrt{g}$ with $g=\left|\operatorname{det} g_{\mu \nu}\right| . \Delta=1$ for a scalar field $\phi$ (3/2 for fermions). To transformation (2.1) one can associate that of a Weyl gauge field $\omega_{\mu}$ which, due to (2.1), has geometric origin:

$$
\omega_{\mu} \rightarrow \omega_{\mu}^{\prime}=\omega_{\mu}-\frac{2}{q} \partial_{\mu} \alpha(x)
$$

Eqs. (2.1), (2.2) define our Weyl (gauge) transformations; $q$ is the coupling to scalar field $\phi$, with Weyl-covariant derivative $\tilde{D}_{\mu} \phi=\left(\partial_{\mu}-q / 2 \omega_{\mu}\right) \phi$. In Weyl geometry $\tilde{\nabla}_{\mu} g_{\alpha \beta}=-q \omega_{\mu} g_{\alpha \beta}$, with $\tilde{\nabla}=d x^{\rho} \tilde{\nabla}_{\rho}$ the Weyl covariant derivative computed with the connection $\tilde{\Gamma}_{\mu \nu}^{\rho}$, where

$$
\tilde{\Gamma}_{\mu \nu}^{\rho}=\Gamma_{\mu \nu}^{\rho}+\frac{q}{2}\left[\delta_{\mu}^{\rho} \omega_{\nu}+\delta_{\nu}^{\rho} \omega_{\mu}-g_{\mu \nu} \omega^{\rho}\right]
$$

\footnotetext{
${ }^{1}$ The conventions used are similar to those in [48], with metric $(+,-,-,-)$.
} 
$\Gamma_{\mu \nu}^{\rho}$ is the Riemannian (Levi-Civita) connection: $\Gamma_{\mu \nu}^{\rho}=(1 / 2) g^{\rho \lambda}\left(\partial_{\mu} g_{\nu \lambda}+\partial_{\nu} g_{\mu \lambda}-\partial_{\lambda} g_{\mu \nu}\right)$. Using eq. (2.3) one verifies that $\left(\tilde{\nabla}_{\mu}+q \omega_{\mu}\right) g_{\alpha \beta}=D_{\mu} g_{\alpha \beta}=0$, with $D_{\mu}$ the Riemannian covariant derivative (computed with $\Gamma_{\mu \nu}^{\rho}$ ). Under eqs. (2.1), (2.2), $\tilde{\Gamma}_{\mu \nu}^{\rho}$ are invariant, which is not true for $\Gamma_{\mu \nu}^{\rho}$. The system is torsion-free (i.e. $\tilde{\Gamma}_{\mu \nu}^{\rho}=\tilde{\Gamma}_{\nu \mu}^{\rho}$ ) and $\tilde{\Gamma}_{\mu \nu}^{\rho} \rightarrow \Gamma_{\mu \nu}^{\rho}$ if $\omega_{\mu} \rightarrow 0$.

Then the Riemann tensor in Weyl geometry, $\tilde{R}_{\mu \nu \sigma}^{\lambda}$, is generated by the new $\tilde{\Gamma}_{\mu \nu}^{\rho}$ by a relation similar to that in the Riemannian geometry:

$$
\tilde{R}_{\mu \nu \sigma}^{\lambda}=\partial_{\nu} \tilde{\Gamma}_{\mu \sigma}^{\lambda}-\partial_{\sigma} \tilde{\Gamma}_{\mu \nu}^{\lambda}+\tilde{\Gamma}_{\nu \rho}^{\lambda} \tilde{\Gamma}_{\mu \sigma}^{\rho}-\tilde{\Gamma}_{\sigma \rho}^{\lambda} \tilde{\Gamma}_{\mu \nu}^{\rho},
$$

and $\tilde{R}_{\mu \sigma}=\tilde{R}_{\mu \lambda \sigma}^{\lambda}, \tilde{R}=g^{\mu \nu} \tilde{R}_{\mu \nu}$. With (2.3), (2.4), $\tilde{R}$ is related to its Riemannian version $(R)$ :

$$
\tilde{R}=R-3 q D_{\mu} \omega^{\mu}-\frac{3}{2} q^{2} \omega^{\mu} \omega_{\mu}
$$

Unlike $R$, under eqs. (2.1), (2.2), $\tilde{R}$ transforms covariantly $\tilde{R}^{\prime}=e^{-2 \alpha(x)} \tilde{R}$, relevant below.

Finally, the Weyl tensor of Weyl geometry $\tilde{C}_{\mu \nu \rho \sigma}$, like its Riemannian counterpart $C_{\mu \nu \rho \sigma}$, is a function of associated Riemann and Ricci tensors and scalar curvature. ${ }^{2}$ Then [22]

$$
\tilde{C}_{\mu \nu \rho \sigma}=C_{\mu \nu \rho \sigma}-\frac{q}{4}\left(g_{\mu \rho} F_{\nu \sigma}+g_{\nu \sigma} F_{\mu \rho}-g_{\mu \sigma} F_{\nu \rho}-g_{\nu \rho} F_{\mu \sigma}\right)+\frac{q}{2} F_{\mu \nu} g_{\rho \sigma},
$$

where $F_{\mu \nu}$ is the field strength of the Weyl gauge field $\omega_{\mu}$; it is given by ${ }^{3} F_{\mu \nu}=\partial_{\mu} \omega_{\nu}-\partial_{\nu} \omega_{\mu}$ and is invariant under transformations (2.1), (2.2), and the same is true for $\sqrt{g} \tilde{C}_{\mu \nu \rho \sigma} \tilde{C}^{\mu \nu \rho \sigma}$.

\subsection{Spontaneous breaking from Weyl gauge symmetry to Einstein gravity}

In the absence of matter, the Weyl action has two quadratic terms, with

$$
L=L_{1}+L_{2}
$$

Here

$$
L_{1}=\sqrt{g} \frac{\xi_{0}}{4 !} \tilde{R}^{2}, \quad \xi_{0}>0 .
$$

Under transformations (2.1), (2.2), $L_{1}$ is invariant. Another invariant under (2.1), (2.2) is

$$
L_{2}=\frac{\sqrt{g}}{\eta} \tilde{C}_{\mu \nu \rho \sigma} \tilde{C}^{\mu \nu \rho \sigma}=\frac{\sqrt{g}}{\eta}\left[C_{\mu \nu \rho \sigma} C^{\mu \nu \rho \sigma}+\frac{3 q^{2}}{2} F_{\mu \nu} F^{\mu \nu}\right]
$$

where $\eta$ is the coupling in Weyl geometry and in the second step we used eq. (2.6). $L_{2}$ is decomposed into the sum of two terms of Riemannian geometry, each of them Weyl invariant. Note the presence of a kinetic term for $\omega_{\mu}$, relevant below. For this term to be canonically normalized in the presence of $L_{2}$, we must set $q^{2}=-\eta / 6$ (so $\left.\eta<0\right)$.

\footnotetext{
${ }^{2}$ Explicitly $\tilde{C}_{\mu \nu \rho \sigma}=\tilde{R}_{\mu \nu \rho \sigma}-(1 / 2)\left(g_{\mu \rho} \tilde{R}_{\nu \sigma}+g_{\nu \sigma} \tilde{R}_{\mu \rho}-g_{\mu \sigma} \tilde{R}_{\nu \rho}-g_{\nu \rho} \tilde{R}_{\mu \sigma}\right)+(1 / 6)\left(g_{\mu \rho} g_{\nu \sigma}-g_{\mu \sigma} g_{\nu \rho}\right) \tilde{R}$. A similar relation exists for the Weyl tensor $C_{\mu \nu \rho \sigma}$ of the Riemannian case, in terms of $R_{\mu \nu \rho \sigma}, R_{\mu \nu}$ and $R$.

${ }^{3}$ In Weyl geometry $\tilde{F}_{\mu \nu}=\tilde{D}_{\mu} \omega_{\nu}-\tilde{D}_{\nu} \omega_{\mu}$ and $\tilde{D}_{\mu} \omega_{\nu}=\partial_{\mu} \omega_{\nu}-\tilde{\Gamma}_{\mu \nu}^{\rho} \omega_{\rho}$. In Riemannian geometry $F_{\mu \nu}=$ $D_{\mu} \omega_{\nu}-D_{\nu} \omega_{\mu}$ and $D_{\mu} \omega_{\nu}=\partial_{\mu} \omega_{\nu}-\Gamma_{\mu \nu}^{\rho} \omega_{\rho}$. Since $\tilde{\Gamma}_{\mu \nu}^{\rho}=\tilde{\Gamma}_{\nu \mu}^{\rho}, \Gamma_{\mu \nu}^{\rho}=\Gamma_{\nu \mu}^{\rho}$, then $\tilde{F}_{\mu \nu}=F_{\mu \nu}=\partial_{\mu} \omega_{\nu}-\partial_{\mu} \omega_{\nu}$.
} 
In the absence of matter fields, $L_{1,2}$ are the only independent terms allowed by Weyl gauge symmetry, up to a topological term that is counterpart to the Riemannian GaussBonnet term and that we do not include here (see e.g. appendix C in [22]).

Returning to $L_{1}, \tilde{R}^{2}$ has higher derivatives, so it propagates an additional scalar state, see e.g. $[49,50]$. We extract from the $\tilde{R}^{2}$ term this (dynamical) degree of freedom via a Lagrangian constraint; this brings a term linear in $\tilde{R}$ and an additional scalar $\phi_{0}$. We have

$$
L_{1}=\sqrt{g} \frac{\xi_{0}}{4 !}\left[-2 \phi_{0}^{2} \tilde{R}-\phi_{0}^{4}\right]
$$

The equation of motion for $\phi_{0}$ gives $\phi_{0}^{2}=-\tilde{R}$. Using this back in (2.10), one recovers $L_{1}$ of (2.8), so Lagrangians (2.8) and (2.10) are classically equivalent. Given its definition, $\phi_{0}$ transforms just like any matter scalar under eq. (2.1). Then a (local) shift symmetry exists, as for a Goldstone (dilaton) field: $\ln \phi_{0}^{2} \rightarrow \ln \phi_{0}^{2}-2 \alpha(x)$. This results from the Weyl invariance $(2.1),(2.2)$ of the term $\phi_{0}^{2} \tilde{R}$; note that its Riemannian counterpart $\left(\phi_{0}^{2} R\right)$ does not have this symmetry, hence the importance of this step (obviously (2.10) is invariant under (2.1), (2.2)).

Using eq. (2.5) to replace $\tilde{R}$ by its Riemannian version $R$ and an integration by parts, then

$$
L_{1}=\sqrt{g}\left[-\frac{\xi_{0}}{12} \phi_{0}^{2} R-\frac{q}{4} g^{\mu \nu} \omega_{\mu} K_{\nu}+\frac{q^{2}}{8} g^{\mu \nu} K \omega_{\mu} \omega_{\nu}-\frac{\xi_{0}}{4 !} \phi_{0}^{4}\right]+\text { total derivative }
$$

where we introduced

$$
K_{\nu}=\partial_{\nu} K, \quad K=\xi_{0} \phi_{0}^{2}
$$

Let us assume first that $L_{1}$ would be the total Lagrangian of our model; its dependence on $\omega_{\mu}$ is algebraic and this field can be integrated out. Its equation of motion is

$$
\omega_{\mu}=\frac{1}{q} \partial_{\mu} \ln K
$$

Inserting this solution back in (2.11) gives

$$
L_{\mathrm{eff}}=\sqrt{g}\left\{-\frac{\xi_{0}}{2}\left[\frac{1}{6} \phi_{0}^{2} R+g^{\mu \nu} \partial_{\mu} \phi_{0} \partial_{\nu} \phi_{0}\right]-\frac{\xi_{0}}{4 !} \phi_{0}^{4}\right\}
$$

$L_{\text {eff }}$ is however uninteresting: although derived from (2.8) and invariant under (2.1), it has a remaining "fake" conformal symmetry $[52,53]$ : its associated current is vanishing. This follows the absence of a kinetic term for the Weyl field which allowed its integration. ${ }^{4,5}$

\footnotetext{
${ }^{4} \mathrm{~A}$ second issue is that with $\xi>0, \phi_{0}$ becomes ghost-like while if $\xi_{0}<0$ and with $\left\langle\phi_{0}\right\rangle \neq 0$, Newton constant would be negative (with our conventions). The Einstein term in our convention is $(-1 / 2) \sqrt{g} M_{p}^{2} R$.

${ }^{5} \mathrm{~A}$ third issue: a conformal transformation (2.1), $\alpha=-\ln \left(6 M_{p} / \xi_{0} \phi\right),\left(M_{p}\right.$ : Planck scale) on (2.14) removes $\phi$ from spectrum and we recover the Einstein action from $L_{\text {eff }}$, but then the number of degrees of freedom in Jordan vs Einstein frame does not match, so "something" is missing; see text after eq. (2.25) for our solution.
} 
This situation is avoided if $\omega_{\mu}$ is dynamical, since then the current does not vanish anymore. A Weyl-invariant kinetic term $\delta L_{2}$ for $\omega_{\mu}$

$$
\delta L_{2}=-\frac{\sqrt{g}}{4} g^{\mu \rho} g^{\nu \sigma} F_{\mu \nu} F_{\rho \sigma}
$$

can be added 'by hand'; but there is no need to do so since $L_{2}$ of (2.9) already contains $\delta L_{2}$ !

To conclude, hereafter we shall consider that the defining action of our model is

$$
L_{1}+\delta L_{2}
$$

If one insists to also include the term $C^{2} \equiv C_{\mu \nu \rho \sigma} C^{\mu \nu \rho \sigma}$, then the action of our model is actually $L_{1}+L_{2}$. In both cases, the equation of motion for $\omega_{\mu}$ gives

$$
\partial^{\alpha}\left(F_{\alpha \mu} \sqrt{g}\right)+\frac{\sqrt{g}}{2} \xi_{0} \phi_{0}\left[\partial_{\mu}-\frac{q}{2} \omega_{\mu}\right] \phi_{0}=0
$$

By applying $\partial^{\mu}$ with $F_{\alpha \mu}$ antisymmetric in $(\alpha, \mu)$, we now find a non-vanishing current

$$
\tilde{K}_{\mu} \equiv \phi_{0}\left(\partial_{\mu}-q / 2 \omega_{\mu}\right) \phi_{0}, \quad \text { with } \quad \partial^{\mu}\left(\sqrt{g} \tilde{K}_{\mu}\right)=0,
$$

so $\tilde{K}_{\mu}$ is conserved. Further, on the ground state, assuming $\phi_{0}(x)=$ constant, it follows that $\partial^{\mu}\left(\sqrt{g} \omega_{\mu}\right)=0$, which is a condition similar to that for a Proca (massive) gauge field, leaving three degrees of freedom for $\omega_{\mu}$. In fact, in the case of a Friedmann-RobertsonWalker (FRW) metric, $g_{\mu \nu}=\left(1,-a^{2}(t),-a^{2}(t),-a^{2}(t)\right)$, with $\phi$ only $t$-dependent, the current conservation (in covariant form $D^{\mu} \tilde{K}_{\mu}=0$ ) leads naturally to $\phi_{0}=$ constant [39].

The other equations of motion, of $g^{\mu \nu}$ (after trace) and of $\phi_{0}$, derived from $L_{1}+\delta L_{2}$, are

$$
\frac{\xi_{0}}{12} \phi_{0}^{2} R+\frac{q}{4} g^{\mu \nu} \omega_{\mu} K_{\nu}-\frac{q^{2}}{8} K \omega^{\mu} w_{\mu}+2 V+\frac{\xi_{0}}{4} \square \phi_{0}^{2}=0
$$

and

$$
-\frac{\xi_{0}}{12} \phi_{0} R+\frac{q^{2}}{8} \omega_{\mu} \omega^{\mu} \xi_{0} \phi_{0}-\frac{1}{2} V^{\prime}+\frac{q}{4 \sqrt{g}} \xi_{0} \phi_{0} \partial_{\mu}\left(\sqrt{g} \omega^{\mu}\right)=0
$$

where we denoted $V \equiv\left(\xi_{0} / 4 !\right) \phi_{0}^{4}$. Eq. (2.20) leads to $\left\langle\phi_{0}^{2}\right\rangle=-\langle\tilde{R}\rangle$ that we already know; thus the ground state has $\tilde{R}=$ constant (this is called Weyl gauge [21]). Further, when adding eqs. (2.19), (2.20), with the last one multiplied by $\phi_{0}$, one finds that on the ground state

$$
4 V\left(\left\langle\phi_{0}\right\rangle\right)-\phi_{0} V^{\prime}\left(\left\langle\phi_{0}\right\rangle\right)=0
$$

The potential is thus a homogeneous function of fields, as expected (given the symmetry); with our $V$, it is automatically respected; $\left\langle\phi_{0}\right\rangle$ is thus a parameter, not fixed by theory; in a Weyl-invariant theory only (dimensionless) ratios of vev's can be fixed.

To see how $\omega_{\mu}$ becomes massive consider a conformal transformation to Einstein frame

$$
\hat{g}_{\mu \nu}=\Omega g_{\mu \nu}, \quad \Omega=\frac{\xi_{0} \phi_{0}^{2}}{6 M^{2}}
$$


$M$ is a mass scale present for dimensional reasons; ${ }^{6}$ its role is discussed shortly. Then

$$
L_{1}=\sqrt{\hat{g}}\left[\frac{-1}{2} M^{2} \hat{R}+\frac{3 M^{2}}{4 \Omega^{2}} \hat{g}^{\mu \nu}\left(\partial_{\mu} \Omega\right)\left(\partial_{\nu} \Omega\right)+\frac{\hat{g}^{\mu \nu}}{\Omega}\left(\frac{-q}{4} \omega_{\mu} K_{\nu}+\frac{q^{2}}{8} K \omega_{\mu} \omega_{\nu}-\frac{\xi_{0}}{4 !} \frac{\phi_{0}^{4}}{\Omega}\right)\right] .
$$

Thus the field $\phi_{0}$ is indeed dynamical, it has a kinetic term. Also note that $\delta L_{2}$ of $(2.15)$ is invariant under (2.22) since the metric part and $F_{\mu \nu}$ are invariant. Further, introduce

$$
\omega_{\mu}^{\prime}=\omega_{\mu}-\frac{1}{q} \partial_{\mu} \ln K
$$

Since $K=\xi_{0} \phi_{0}^{2}$ then $\phi_{0}$ is absorbed into $\omega_{\mu}^{\prime}$ in (2.24) where also $\partial_{\mu} \ln K=\partial_{\mu} \ln \Omega$. Using (2.24), replace $\omega_{\mu}$ in $L_{1}$ and denote by $F_{\mu \nu}^{\prime}$ the field strength of $\omega_{\mu}^{\prime}$. Then the total Lagrangian is

$$
L_{1}+\delta L_{2}=\sqrt{\hat{g}}\left[-\frac{1}{2} M^{2} \hat{R}-\frac{3 M^{4}}{2 \xi_{0}}\right]+\sqrt{\hat{g}}\left[-\frac{1}{4} \hat{g}^{\mu \rho} \hat{g}^{\nu \sigma} F_{\mu \nu}^{\prime} F_{\rho \sigma}^{\prime}+\frac{3 q^{2}}{4} M^{2} \hat{g}^{\mu \nu} \omega_{\mu}^{\prime} w_{\nu}^{\prime}\right] .
$$

It is important to note that there is no kinetic term left for $\phi_{0}$, since it was cancelled by that generated when expressing $\omega_{\mu}$-dependence in eq. (2.23) in terms of $\omega_{\mu}^{\prime}$. The massless Weyl field has become massive after "eating" the Goldstone mode $\left(\phi_{0}\right)$, via a Stueckelberg mechanism, without a corresponding Higgs mode in the spectrum or a potential. This mechanism essentially re-distributes the degrees of freedom in the action: the initial massless $\omega_{\mu}$ and the real scalar $\phi_{0}$ are converted into a single massive Weyl field $\omega_{\mu}$ with three degrees of freedom (recall $\partial^{\mu}\left(\sqrt{g} \omega_{\mu}\right)=0$ ); so the number of degrees of freedom is indeed conserved when going from the Jordan to the Einstein frame (as it should).

Having checked this conservation, eqs. (2.22), (2.24) may also be seen as a particular Weyl transformation eqs. (2.1), (2.2) of $2 \alpha(x)=\ln \Omega$, to the unitary gauge ("gauge fixing") where the Goldstone $\phi_{0}$ is absent (having been "eaten" by now massive $\omega_{\mu}^{\prime}$ ) and giving $\hat{\phi}_{0}^{2}=\phi_{0}^{2} / \Omega=6 M^{2} / \xi_{0}=$ constant. The unitary gauge being non-renormalizable, one should not use (2.25) for loop calculations, but use instead e.g. (2.11), (2.15).

In eq. (2.25) we obtained the Einstein-Hilbert action, a positive cosmological constant and the Proca action for a massive Weyl field ${ }^{7} \omega_{\mu}^{\prime}$; its mass is related to the Planck scale $\left(M_{p}\right)$

$$
M_{p}^{2}=M^{2}, \quad m_{\omega}^{2}=(3 / 2) q^{2} M^{2} .
$$

The value of $m_{\omega}$ depends on the gauge coupling $q$ in $L_{1}+\delta L_{2}$, see $L_{1}$ of (2.11) and canonically normalised $\delta L_{2}$ of (2.15). Below the scale $m_{\omega}$ the Weyl field decouples and Einstein gravity is obtained as a "low-energy" effective theory limit. The scale $M$ introduced on dimensional grounds in (2.22), remains undetermined by the theory. From (2.11),

\footnotetext{
${ }^{6}$ One may avoid introducing $M$ if $\hat{g}_{\mu \nu}$ is dimensionful [26], which may be acceptable since the metric is a dynamical variable of kinetic term found in $\tilde{C}_{\mu \nu \rho \sigma}^{2}=C_{\mu \nu \rho \sigma}^{2}+\ldots$ rather than a simple dimensionless constant.

${ }^{7}$ Our above result is consistent with those in [49-51] where it was shown that 'pure' $R^{2}$ gravity in the Riemannian geometry, describes Einstein gravity plus a cosmological constant and a scalar (Goldstone) field. In our case the Goldstone mode is eaten by the Weyl gauge field present in the $\tilde{R}^{2}$-term in Weyl geometry.
} 
$M^{2}=\xi_{0}\left\langle\phi_{0}\right\rangle^{2} / 6$ which is equally undetermined in a theory invariant under (2.1), (2.2), as discussed. ${ }^{8}$

In the decoupling limit of the massive Weyl gauge field, Weyl geometry "flows" into a Riemannian geometry. This may also be seen dynamically from the conserved current in (2.18) which for a FRW metric is driving $\phi_{0}$ to a constant value [39] (in this case $\propto M$ ). Below $m_{\omega}$ the Weyl gauge field is absent, therefore the Weyl connection becomes that of the Riemannian geometry, $\tilde{\Gamma}_{\mu \nu}^{\rho}=\Gamma_{\mu \nu}^{\rho}$. As a result, long-held criticisms of Weyl quadratic gravity without matter, such as (see e.g. [2-4, 21]): the changing of the norm of a vector under parallel transport on a closed curve, or the changing of the atomic spectral lines, do not apply since such effects are strongly suppressed by a high scale $m_{\omega} \propto M_{p}$.

So far our analysis was based on the Lagrangian $L_{1}+\delta L_{2}$. Considering instead the Lagrangian $L=L_{1}+L_{2}$, one has to include the remaining term in the r.h.s. of (2.9), i.e. $C^{2} \equiv C_{\mu \nu \rho \sigma} C^{\mu \nu \rho \sigma}$; this is immediate, since this is invariant under (2.22), (2.24). This term provides the kinetic term for the metric [27] and is needed at the quantum level to renormalize divergences like $k^{4}$. However, in this case the coupling $q$ cannot be adjusted at will anymore, being proportional to $\eta$ (see text after (2.9)). Lowering $q$ too much brings a too light mass $m_{g}^{2} \sim \eta M^{2}$ of the spin-two ghost of the $C^{2}$ term, together with its instability.

It is interesting that Lagrangian (2.8), (2.9) dictated by Weyl geometry (no matter) is so rich in structure, encoding Stueckelberg mechanism, dilaton $\phi_{0}$, Einstein action, Proca action for massive $\omega_{\mu}$, a positive cosmological constant and fields kinetic terms and interactions.

\section{Adding matter fields}

\subsection{Spontaneous breaking of Weyl gauge symmetry}

Let us now consider the SM scalar sector in addition to Weyl quadratic gravity action, with all dimension-four couplings allowed by the Weyl gauge symmetry. Note that the SM fermions do not have couplings to the Weyl gauge field (in the absence of torsion) $[13,15]$. We consider the SM Higgs field and denote by $\phi_{1}$ its neutral component. Then the Lagrangian we study, invariant under $(2.1),(2.2)$, and written in a Weyl geometry language, is

$$
\mathcal{L}=\left(L_{1}+\delta L_{2}\right)+\sqrt{g}\left[-\frac{1}{12} \xi_{1} \phi_{1}^{2} \tilde{R}+\frac{1}{2} g^{\mu \nu} \tilde{D}_{\mu} \phi_{1} \tilde{D}_{\nu} \phi_{1}-V_{1}\right]
$$

with Weyl-covariant derivative $\tilde{D}_{\mu} \phi_{1}=\left(\partial_{\mu}-q / 2 \omega_{\mu}\right) \phi_{1}$ and $L_{1}$ and $\delta L_{2}$ of eqs. (2.8), (2.15)

$$
L_{1}+\delta L_{2}=\sqrt{g} \frac{\xi_{0}}{4 !} \tilde{R}^{2}-\frac{\sqrt{g}}{4} g^{\mu \rho} g^{\nu \sigma} F_{\mu \nu} F_{\rho \sigma} .
$$

The Weyl tensor squared term $C_{\mu \nu \rho \sigma} C^{\mu \nu \rho \sigma}$ in $L_{2}$ of (2.9) is not included in $\mathcal{L}$, but since it is not affecting the transformations below, it can easily be added (replace $\delta L_{2} \rightarrow L_{2}$ ). Also $F_{\mu \nu}$ is the same in both Riemann and Weyl geometry (in the absence of torsion, as here).

\footnotetext{
${ }^{8}$ In conformal theory only ratios of scales can be predicted in terms of dimensionless couplings. If this symmetry is broken explicitly (by quantum corrections) dimensional transmutation can determine a field vev.
} 
The only possible form of the Higgs potential $V_{1}$ consistent with the symmetry, is

$$
V_{1}=\frac{\lambda_{1}}{4 !} \phi_{1}^{4}
$$

Using (2.10) and (2.5), one finds, following steps similar to the previous section ${ }^{9}$

$$
\mathcal{L}=\delta L_{2}+\sqrt{g}\left[-\frac{1}{12} \xi_{a} \phi_{a}^{2} R-\frac{q}{4} g^{\mu \nu} \omega_{\mu} \partial_{\nu} \mathcal{K}+\frac{q^{2}}{8} g^{\mu \nu} \mathcal{K} \omega_{\mu} \omega_{\nu}+\frac{1}{2} g^{\mu \nu} \partial_{\mu} \phi_{1} \partial_{\nu} \phi_{1}-\mathcal{V}\left(\phi_{0}, \phi_{1}\right)\right]
$$

up to a total derivative, with

$$
\begin{aligned}
\mathcal{V} & =\frac{\xi_{0}}{4 !} \phi_{0}^{4}+\frac{\lambda_{1}}{4 !} \phi_{1}^{4}, \\
\mathcal{K}_{\mu} & =\partial_{\mu} \mathcal{K}, \quad \mathcal{K}=\xi_{a} \phi_{a}^{2}+\phi_{1}^{2}, \quad \text { sum over } a=0,1 .
\end{aligned}
$$

In (3.4) a sum over the repeated index "a" is understood, with $a=0,1$. The generalisation of this action to more matter (scalar) fields is immediate.

We perform a conformal transformation to the Einstein frame, to a new metric $\hat{g}_{\mu \nu}$

$$
\hat{g}_{\mu \nu}=\Omega g_{\mu \nu}, \quad \Omega=\frac{1}{6 M^{2}}\left(\xi_{0} \phi_{0}^{2}+\xi_{1} \phi_{1}^{2}\right)
$$

One has

$$
\begin{aligned}
\mathcal{L}= & \sqrt{\hat{g}}\left\{-\frac{1}{2} M^{2} \hat{R}+\frac{3}{4} \hat{g}^{\mu \nu} M^{2} \partial_{\mu} \ln \Omega \partial_{\nu} \ln \Omega\right. \\
& \left.+\frac{\hat{g}^{\mu \nu}}{\Omega}\left[\frac{1}{2} \partial_{\mu} \phi_{1} \partial_{\nu} \phi_{1}-\frac{q}{4} \omega_{\mu} \partial_{\nu} \mathcal{K}+\frac{q^{2}}{8} \mathcal{K} \omega_{\mu} \omega^{\mu}\right]-\frac{1}{4} \hat{g}^{\mu \rho} \hat{g}^{\nu \sigma} F_{\mu \nu} F_{\rho \sigma}-\frac{\mathcal{V}}{\Omega^{2}}\right\}
\end{aligned}
$$

Further, introduce

$$
\omega_{\mu}^{\prime}=\omega_{\mu}-\frac{1}{q} \partial_{\mu} \ln \mathcal{K}
$$

The kinetic terms in $\mathcal{L}$ for $\phi_{0}$ and $\phi_{1}$ (hereafter $\mathcal{L}_{\text {k.t. }}$ ) become, after using eqs. (3.7), (3.9)

$$
\begin{aligned}
\mathcal{L}_{\text {k.t. }} & =\sqrt{\hat{g}} \hat{g}^{\mu \nu} \frac{1}{8 \Omega}\left[\frac{6 M^{2}}{\Omega}\left(\partial_{\mu} \Omega\right)\left(\partial_{\nu} \Omega\right)-\frac{1}{\mathcal{K}}\left(\partial_{\mu} \mathcal{K}\right)\left(\partial_{\nu} \mathcal{K}\right)+4\left(\partial_{\mu} \phi_{1}\right)\left(\partial_{\nu} \phi_{1}\right)\right] \\
& =\frac{3 M^{2}}{4} \frac{\sqrt{\hat{g}} \hat{g}^{\mu \nu}}{1+\mathcal{Z}}\left(\partial_{\mu} \ln \mathcal{Z}\right)\left(\partial_{\nu} \ln \mathcal{Z}\right), \quad \text { with } \quad \mathcal{Z} \equiv \xi_{0} \frac{\phi_{0}^{2}}{\phi_{1}^{2}}+\xi_{1} .
\end{aligned}
$$

We see there is only one kinetic term left in the action, for the new variable $\mathcal{Z}$ which is a combination of initial $\phi_{0}, \phi_{1}$. One could introduce polar coordinates fields $(\rho, \theta)$, such as

\footnotetext{
${ }^{9}$ In $V_{1}$ there is no classical coupling of $\phi_{1}$ to the dilaton $\left(\phi_{0}\right)$ hidden in Weyl's quadratic action (3.2) since $\phi_{0}$ is an intrinsic part of our $\tilde{R}^{2}$ term from which is extracted by "linearisation" of $\tilde{R}^{2}$, eq. (2.10). Adding to $V_{1}$ a term $\left(\lambda_{m} / 12\right) \phi_{0}^{2} \phi_{1}^{2}$ is also redundant, since together with (2.10), integrating out $\phi_{0}$ simply restores the original $\tilde{R}^{2}$ term after a redefinition of initial couplings of $\phi_{1}: \lambda_{1} \rightarrow \lambda_{1}+\lambda_{m}^{2} / \xi_{0}, \quad \xi_{1} \rightarrow \xi_{1}+\lambda_{m}$. Also, adding a term $\tilde{\xi} \phi_{0}^{2} \tilde{R}$ to (3.2) would introduce an extra (dynamical) degree of freedom beyond the dilaton in $\tilde{R}^{2}$ term!
} 
$\phi_{0}=\left(1 / \sqrt{\xi}_{0}\right) \rho \sin \theta$ and $\phi_{1}=\left(1 / \sqrt{1+\xi_{1}}\right) \rho \cos \theta$. In such basis $\mathcal{Z}$ is an "angular" variable field while $\mathcal{K}$ entering in (3.9) becomes $\mathcal{K}=\rho^{2}$ and is the "radial" direction in field space.

After transformation (3.9) the terms ${ }^{10}$ in (3.8) other than $\mathcal{L}_{\text {k.t. }}$ also depend only on the ratio $\phi_{0} / \phi_{1} \sim \mathcal{Z}$ and not on the radial direction field! To anticipate, this is explained by $\omega_{\mu}^{\prime}$ that must have "eaten" the radial direction in (3.9) (Stueckelberg mechanism), see later.

We bring to canonical form the kinetic term $\mathcal{L}_{\text {k.t. }}$ by replacing

$$
\frac{1}{\mathcal{Z}}=\sinh ^{2} \frac{h}{M \sqrt{6}}
$$

where $h$ is our (neutral) Higgs field. This gives that $\mathcal{L}_{\text {k.t. }}=(1 / 2) \sqrt{\hat{g}} \hat{g}^{\mu \nu}\left(\partial_{\mu} h\right)\left(\partial_{\nu} h\right)$.

From $\mathcal{L}$ of (3.8), using (3.11) and notations (3.9), (3.12), we obtain our final Lagrangian

$$
\mathcal{L}=\sqrt{\hat{g}}\left[-\frac{M^{2}}{2} \hat{R}+\frac{\hat{g}^{\mu \nu}}{2}\left(\partial_{\mu} h\right)\left(\partial_{\nu} h\right)-\frac{1}{4} \hat{g}^{\mu \rho} \hat{g}^{\nu \sigma} F_{\mu \nu}^{\prime} F_{\rho \sigma}^{\prime}+\frac{m_{\omega}^{2}(h)}{2} \omega_{\mu}^{\prime} \omega^{\prime \mu}-\hat{\mathcal{V}}(h)\right],
$$

where $F_{\mu \nu}^{\prime}$ is the field strength of $\omega_{\mu}^{\prime}$ and

$$
\begin{aligned}
m_{\omega}^{2}(h) & =\frac{q^{2} \mathcal{K}}{4 \Omega}=\frac{3}{2} M^{2} q^{2} \cosh ^{2} \frac{h}{M \sqrt{6}}, \\
\hat{\mathcal{V}}(h) & =\frac{\mathcal{V}}{\Omega^{2}}=\frac{3}{2} \frac{M^{4}}{\xi_{0}}\left[1-2 \xi_{1} \sinh ^{2} \frac{h}{M \sqrt{6}}+\left(\lambda_{1} \xi_{0}+\xi_{1}^{2}\right) \sinh ^{4} \frac{h}{M \sqrt{6}}\right] .
\end{aligned}
$$

For small higgs field values $h \ll M$ one has

$$
\begin{aligned}
m_{\omega}^{2}(h) & =\frac{3}{2} M^{2} q^{2}\left[1+\frac{h^{2}}{6 M^{2}}+\frac{h^{4}}{108 M^{4}}+\mathcal{O}\left(h^{6} / M^{6}\right)\right] \\
\hat{\mathcal{V}}(h) & =\frac{3 M^{4}}{2 \xi_{0}}-\frac{\xi_{1} M^{2}}{2 \xi_{0}} h^{2}+\frac{1}{4 !}\left[\lambda_{1}+\frac{\xi_{1}\left(3 \xi_{1}-2\right)}{3 \xi_{0}}\right] h^{4}+\mathcal{O}\left(h^{6} / M^{6}\right)
\end{aligned}
$$

The first term on the r.h.s. of (3.16) is the mass of the Weyl gauge field $\omega_{\mu}^{\prime}$, up to additional corrections of order $\mathcal{O}\left(\langle h\rangle^{2} / M^{2}\right)$ due to the Higgs mechanism itself, if $\langle h\rangle \neq 0$ (see below). Therefore, following eq. (3.9) the "radial" degree of freedom in the field space (dilaton) was "eaten" by the gauge field $\omega_{\mu}^{\prime}$ which has become massive, via the Stueckelberg mechanism. The number of degrees of freedom is conserved: initially we had 2 real scalars $\left(\phi_{0}, \phi_{1}\right)$ and massless $\omega_{\mu}$ which were re-arranged into one real scalar $h$ and a massive gauge field $\omega_{\mu}^{\prime}$.

$\mathcal{L}$ in eq. (3.13) includes the Einstein action with a positive cosmological constant, a massive Weyl gauge field and a potential for the Higgs field $h$. For a vanishing non-minimal coupling $\xi_{1}=0$, see our starting $\mathcal{L}$ in $(3.1)$, one recovers the initial potential for $\phi_{1}$ plus a cosmological constant similar to that in Weyl quadratic action without matter, eq. (2.25).

Eqs. (3.13) to (3.15) give the Higgs sector for the SM enlarged with Weyl gauge symmetry and can be used for further investigations of this symmetry. These equations bring no restrictions at the classical level for the value of $h$ relative to (otherwise arbitrary) $M$.

\footnotetext{
${ }^{10}$ These are the terms in (3.8) proportional to $\mathcal{V} / \Omega^{2}$ and $\mathcal{K} / \Omega$ and clearly depend only of the ratio $\phi_{0} / \phi_{1}$.
} 


\subsection{Electroweak symmetry breaking}

From (3.15), (3.17), we see that a non-minimal coupling $\xi_{1} \tilde{R}^{2} \phi_{1}^{2},\left(\xi_{1}>0\right)$ induced a negative quadratic term in the potential and spontaneous electroweak symmetry breaking, with

$$
m_{h}^{2}=\frac{\xi_{1}}{\xi_{0}} M^{2} .
$$

A hierarchy $m_{h}^{2} \ll M^{2}$ can be arranged by a classical tuning $\xi_{1} \ll \xi_{0}$ to an ultraweak value of $\xi_{1}$. This is a gravitational higgs mechanism (which forbids the presence of TeV-scale squarks!). ${ }^{11}$

Regarding the scale $M$, in the limit $h \ll M$, can be identified with a constant value of the dilaton $\left\langle\phi_{0}\right\rangle$ (in this limit $\left\langle\phi_{0}\right\rangle \gg\left\langle\phi_{1}\right\rangle$ ); like $M,\left\langle\phi_{0}\right\rangle$ is a parameter not fixed by the theory and must be tuned to the actual Planck scale value (as mentioned, only ratios of scales can be determined in a Weyl invariant theory). Finally, the vacuum energy is still positive, dominated by the dilaton contribution $\hat{\mathcal{V}}_{\text {min }}=3 M^{4} /\left(2 \xi_{0}\right)\left[1-\xi_{1}^{2} /\left(\xi_{0} \lambda_{1}\right)+\ldots\right]$.

A study of the quantum corrections to the Higgs mass is beyond the goal of this paper. However, we stress here the role Weyl gauge symmetry may play at the quantum level. Note that classically only the Higgs sector of the SM couples to the Weyl field $\omega_{\mu}[13,15]$. Using a Weyl-invariant regularization [47] one could answer whether Weyl gauge symmetry can protect $\xi_{1}$ and thus the Higgs mass $m_{h} \propto \xi_{1}$ against large quantum corrections. ${ }^{12}$ Note that each of the terms in our $\mathcal{L}$ of (3.1), (3.2) is separately Weyl gauge invariant, see eqs. (2.1), (2.2). We expect that this symmetry bring some "protection" for the ultraviolet (UV) behaviour of this theory. In particular we expect a better UV behaviour than in Riemannian gravity e.g. Agravity [44, 45] where no similar local symmetry (Weyl, conformal) exists. This motivates a quantum analysis of Weyl quadratic gravity.

In the light of the results in [46] for renormalizability, one would expect a Weyl quadratic theory given by $L_{1}+L_{2}$ of (2.8), (2.9) be renormalizable. One must however pay attention to the analytical continuation from Minkowski to the Euclidean space which is non-trivial in the presence of higher derivative terms [54].

\section{Conclusions}

We considered the general action of Weyl gravity in the absence of matter, which is the sum of two terms quadratic in the curvature scalar $(\tilde{R})$ and in the Weyl tensor $\left(\tilde{C}_{\mu \nu \rho \sigma}\right)$ of the Weyl conformal geometry, then studied its spontaneous breaking. We also studied the effect of coupling Weyl (quadratic) gravity to a Higgs-like matter sector.

In the absence of matter fields, the Weyl gauge field $\omega_{\mu}$ in the action becomes massive, with a mass $m_{\omega} \sim q M_{p}$ where $q$ is the coupling and $M_{p}$ the Planck scale. This happens via a Stueckelberg mechanism which is essentially a re-arrangement of the degrees of freedom (without a higgs vev or potential needed): the field $\omega_{\mu}$ "eats" a scalar degree of freedom

\footnotetext{
${ }^{11}$ The quartic term in (3.17) remains positive as long as $3 \lambda_{1} \xi_{0}>\left(2-3 \xi_{1}\right) \xi_{1}\left(\lambda_{1}>0, \xi_{1}>0\right)$ which is easily satisfied for small enough $\xi_{1} \ll \xi_{0}$ required for small Higgs mass. Also note from eq. (3.15) that at large $h$, the quartic term receives overall positive corrections from the gravitational effects, proportional to $\xi_{1}^{2} / \xi_{0}$.

${ }^{12}$ Without additional fine tuning beyond the classical one mentioned above.
} 
(dilaton) "extracted" from the $\tilde{R}^{2}$ term. The necessary presence of a kinetic term for the Weyl gauge field originates from the term $\tilde{C}_{\mu \nu \rho \sigma} \tilde{C}^{\mu \nu \rho \sigma}$ of Weyl geometry. However, if this is not included in the initial Weyl quadratic action, the gauge kinetic term may be added on its own in Weyl geometry, on symmetry arguments only.

After the Stueckelberg mechanism, one obtains the Einstein-Hilbert action, with a positive cosmological constant and the Proca action for the massive Weyl gauge field (and a Riemannian Weyl-tensor-squared term, if $\tilde{C}_{\mu \nu \rho \sigma} \tilde{C}^{\mu \nu \rho \sigma}$ was included initially). No additional matter scalar field (Higgs, etc.) is needed to this purpose. Below the mass $m_{\omega}$, the Weyl field $\omega_{\mu}$ decouples and Weyl geometry (connection) becomes Riemannian. Therefore, the Einstein-Hilbert action is a "low-energy" effective theory limit of the Weyl quadratic gravity (without matter) which avoids in this way previous, long-held criticisms against it.

This result has consequences for physics at high scale where Weyl gauge symmetry may be present (inflation, black hole physics, conformal supergravity). During the spontaneous breaking of this symmetry the number of degrees of freedom is indeed conserved, also when going to the unitary gauge $\hat{\phi}_{0}=$ constant (unlike in models invariant under "usual" Weyl symmetry, eq. (2.1)). It is remarkable that the simple Weyl quadratic action dictated by Weyl geometry alone is so rich in structure, encoding a Stueckelberg mechanism, the Einstein-Hilbert action, Proca action, positive cosmological constant, the dilaton, the metric and their interactions. Further study of this symmetry should use the Weyl geometry formulation which is easier (than the Riemannian one) since then the scalar curvature transforms covariantly, so each operator respects this symmetry. This places on equal footing, in the Lagrangian, Weyl gauge symmetry and (internal) gauge symmetries.

In the presence of a scalar matter field $\phi_{1}$ (Higgs), the Weyl gauge symmetry allows a non-minimal coupling $\xi_{1} \phi_{1}^{2} \tilde{R}$, in addition to the mentioned Weyl quadratic action and to the matter action; the latter is that of the SM Higgs sector with a potential $\lambda_{1} \phi_{1}^{4} / 4$ ! (as the only one allowed by this symmetry). The Stueckelberg breaking mechanism is still at work and the Weyl gauge field is "eating" the dilaton (the radial direction in the field space of $\phi_{0}$, $\left.\phi_{1}\right)$ which subsequently disappears from the action. At the same time, in the Riemannian limit ( $\omega_{\mu}$ decoupled), the scalar potential of the remaining Higgs degree of freedom acquires at low energy $(h \ll M)$ a negative quadratic term $\propto \xi_{1} / \xi_{0}$. This is gravitationally-induced spontaneous electroweak symmetry breaking. It is worth investigating further if the Higgs mass value $\left(\xi_{1} / \xi_{0}\right.$ in Planck units) is stable at the quantum level in SM with spontaneously broken Weyl gauge symmetry.

Note added. After completing this work we became aware of [55-57] where the connection in a general theory of gravity is shown to acquire mass via a Higgs mechanism, while at low scales is "frozen" to the Levi-Civita connection. This is consistent with our result.

\section{Acknowledgments}

We thank Mihai Visinescu (IFIN Bucharest) and Hyun Min Lee (Chung-Ang University, Seoul) for discussions on Weyl geometry and its applications. 
Open Access. This article is distributed under the terms of the Creative Commons Attribution License (CC-BY 4.0), which permits any use, distribution and reproduction in any medium, provided the original author(s) and source are credited.

\section{References}

[1] H. Weyl, Gravitation und Elektrizität, Sitzungsberichte der Königlich Preussischen Akademie der Wissenschaften zu Berlin (1918), pp.465-480, Space-time-matter, translated from German by H.L. Brose, Methuen \& Co Ltd, London (1922).

[2] E. Scholz, The unexpected resurgence of Weyl geometry in late 20-th century physics, Einstein Stud. 14 (2018) 261 [arXiv:1703.03187].

[3] E. Scholz, Paving the Way for Transitions - A Case for Weyl Geometry, Einstein Stud. 13 (2017) 171 [arXiv:1206.1559].

[4] E. Scholz, Weyl geometry in late 20th century physics, arXiv:1111.3220 [INSPIRE].

[5] P.A.M. Dirac, Long range forces and broken symmetries, Proc. Roy. Soc. Lond. A 333 (1973) 403.

[6] C. Brans and R.H. Dicke, Mach's principle and a relativistic theory of gravitation, Phys. Rev. 124 (1961) 925 [INSPIRE].

[7] R.H. Dicke, Mach's principle and invariance under transformation of units, Phys. Rev. 125 (1962) 2163 [INSPIRE].

[8] P. Jordan, Schwerkraft und Weltall, (1952), Braunschweig, Vieweg, 2nd revised edition (1955).

[9] L. Smolin, Towards a Theory of Space-Time Structure at Very Short Distances, Nucl. Phys. B 160 (1979) 253 [INSPIRE].

[10] H. Cheng, The Possible Existence of Weyl's Vector Meson, Phys. Rev. Lett. 61 (1988) 2182 [INSPIRE].

[11] I. Quiros, Scale invariant theory of gravity and the standard model of particles, arXiv: 1401.2643 [INSPIRE].

[12] J.T. Wheeler, Weyl geometry, Gen. Rel. Grav. 50 (2018) 80 [arXiv:1801.03178] [InSPIRE].

[13] M. de Cesare, J.W. Moffat and M. Sakellariadou, Local conformal symmetry in non-Riemannian geometry and the origin of physical scales, Eur. Phys. J. C 77 (2017) 605 [arXiv: 1612.08066] [INSPIRE].

[14] H.C. Ohanian, Weyl gauge-vector and complex dilaton scalar for conformal symmetry and its breaking, Gen. Rel. Grav. 48 (2016) 25 [arXiv:1502.00020] [INSPIRE].

[15] D.M. Ghilencea and H.M. Lee, Weyl gauge symmetry and its spontaneous breaking in Standard Model and inflation, arXiv:1809.09174 [INSPIRE].

[16] A. Barnaveli, S. Lucat and T. Prokopec, Inflation as a spontaneous symmetry breaking of Weyl symmetry, JCAP 01 (2019) 022 [arXiv: 1809.10586] [INSPIRE].

[17] J.W. Moffat, Scalar-tensor-vector gravity theory, JCAP 03 (2006) 004 [gr-qc/0506021] [INSPIRE].

[18] L. Heisenberg, Scalar-Vector-Tensor Gravity Theories, JCAP 10 (2018) 054 [arXiv: 1801.01523] [INSPIRE]. 
[19] J. Beltran Jimenez, L. Heisenberg and T.S. Koivisto, Cosmology for quadratic gravity in generalized Weyl geometry, JCAP 04 (2016) 046 [arXiv: 1602.07287] [INSPIRE].

[20] C.T. Hill, Inertial Symmetry Breaking, in Proceedings, 11th International Workshop on the Physics of Excited Nucleons (NSTAR 2017), Columbia, SC, U.S.A., August 20-23, 2017 (2018) [arXiv: 1803.06994] [INSPIRE].

[21] E. Scholz, Higgs and gravitational scalar fields together induce Weyl gauge, Gen. Rel. Grav. 47 (2015) 7 [arXiv: 1407.6811] [inSPIRE].

[22] W. Drechsler and H. Tann, Broken Weyl invariance and the origin of mass, Found. Phys. 29 (1999) 1023 [gr-qc/9802044] [INSPIRE].

[23] E.C.G. Stueckelberg, Interaction forces in electrodynamics and in the field theory of nuclear forces, Helv. Phys. Acta 11 (1938) 299 [inSPIRE].

[24] G. 't Hooft, Local Conformal Symmetry: the Missing Symmetry Component for Space and Time, arXiv:1410.6675 [INSPIRE].

[25] I. Bars, P. Steinhardt and N. Turok, Local Conformal Symmetry in Physics and Cosmology, Phys. Rev. D 89 (2014) 043515 [arXiv:1307.1848] [InSPIRE].

[26] G. 't Hooft, Imagining the future, or how the Standard Model may survive the attacks, Int. J. Mod. Phys. 31 (2016) 1630022 [INSPIRE].

[27] G. 't Hooft, Local conformal symmetry in black holes, standard model and quantum gravity, Int. J. Mod. Phys. D 26 (2016) 1730006 [inSPIRE].

[28] M. Shaposhnikov and D. Zenhausern, Quantum scale invariance, cosmological constant and hierarchy problem, Phys. Lett. B 671 (2009) 162 [arXiv:0809.3406] [INSPIRE].

[29] R. Armillis, A. Monin and M. Shaposhnikov, Spontaneously Broken Conformal Symmetry: Dealing with the Trace Anomaly, JHEP 10 (2013) 030 [arXiv:1302.5619] [INSPIRE].

[30] F. Bezrukov, G.K. Karananas, J. Rubio and M. Shaposhnikov, Higgs-Dilaton Cosmology: an effective field theory approach, Phys. Rev. D 87 (2013) 096001 [arXiv:1212.4148] [INSPIRE].

[31] F. Gretsch and A. Monin, Perturbative conformal symmetry and dilaton, Phys. Rev. D 92 (2015) 045036 [arXiv: 1308.3863] [INSPIRE].

[32] D.M. Ghilencea, Quantum implications of a scale invariant regularization, Phys. Rev. D 97 (2018) 075015 [arXiv: 1712.06024] [INSPIRE].

[33] D.M. Ghilencea, Manifestly scale-invariant regularization and quantum effective operators, Phys. Rev. D 93 (2016) 105006 [arXiv:1508.00595] [INSPIRE].

[34] D.M. Ghilencea, One-loop potential with scale invariance and effective operators, PoS (CORFU2015) 040 (2016) [arXiv: 1605.05632] [INSPIRE].

[35] D.M. Ghilencea, Z. Lalak and P. Olszewski, Two-loop scale-invariant scalar potential and quantum effective operators, Eur. Phys. J. C 76 (2016) 656 [arXiv:1608.05336] [InSPIRE].

[36] D.M. Ghilencea, Z. Lalak and P. Olszewski, Standard Model with spontaneously broken quantum scale invariance, Phys. Rev. D 96 (2017) 055034 [arXiv: 1612.09120] [INSPIRE].

[37] R. Foot, A. Kobakhidze, K.L. McDonald and R.R. Volkas, Poincaré protection for a natural electroweak scale, Phys. Rev. D 89 (2014) 115018 [arXiv:1310.0223] [INSPIRE].

[38] P.G. Ferreira, C.T. Hill and G.G. Ross, Scale-Independent Inflation and Hierarchy Generation, Phys. Lett. B 763 (2016) 174 [arXiv:1603.05983] [INSPIRE]. 
[39] P.G. Ferreira, C.T. Hill and G.G. Ross, Inertial Spontaneous Symmetry Breaking and Quantum Scale Invariance, Phys. Rev. D 98 (2018) 116012 [arXiv:1801.07676] [InSPIRE].

[40] P.G. Ferreira, C.T. Hill and G.G. Ross, Weyl Current, Scale-Invariant Inflation and Planck Scale Generation, Phys. Rev. D 95 (2017) 043507 [arXiv: 1610.09243] [INSPIRE].

[41] E.J. Chun, S. Jung and H.M. Lee, Radiative generation of the Higgs potential, Phys. Lett. B $\mathbf{7 2 5}$ (2013) 158 [Erratum ibid. B $\mathbf{7 3 0}$ (2014) 357] [arXiv: 1304.5815] [INSPIRE].

[42] O. Lebedev and H.M. Lee, Higgs Portal Inflation, Eur. Phys. J. C 71 (2011) 1821 [arXiv: 1105.2284] [INSPIRE].

[43] Z. Lalak and P. Olszewski, Vanishing trace anomaly in flat spacetime, Phys. Rev. D 98 (2018) 085001 [arXiv:1807.09296] [INSPIRE].

[44] A. Salvio and A. Strumia, Agravity, JHEP 06 (2014) 080 [arXiv:1403.4226] [InSPIRE].

[45] A. Salvio and A. Strumia, Agravity up to infinite energy, Eur. Phys. J. C 78 (2018) 124 [arXiv: 1705. 03896] [INSPIRE].

[46] K.S. Stelle, Renormalization of Higher Derivative Quantum Gravity, Phys. Rev. D 16 (1977) 953 [INSPIRE].

[47] F. Englert, C. Truffin and R. Gastmans, Conformal Invariance in Quantum Gravity, Nucl. Phys. B 117 (1976) 407 [INSPIRE].

[48] D. Gorbunov and V. Rubakov, Introduction to the theory of the early Universe, World Scientific (2011).

[49] C. Kounnas, D. Lüst and N. Toumbas, $R^{2}$ inflation from scale invariant supergravity and anomaly free superstrings with fluxes, Fortsch. Phys. 63 (2015) 12 [arXiv:1409.7076] [INSPIRE].

[50] L. Álvarez-Gaumé, A. Kehagias, C. Kounnas, D. Lüst and A. Riotto, Aspects of Quadratic Gravity, Fortsch. Phys. 64 (2016) 176 [arXiv: 1505.07657] [INSPIRE].

[51] A. Edery and Y. Nakayama, Gravitating magnetic monopole via the spontaneous symmetry breaking of pure $R^{2}$ gravity, Phys. Rev. D 98 (2018) 064011 [arXiv:1807.07004] [INSPIRE].

[52] R. Jackiw and S.-Y. Pi, Fake Conformal Symmetry in Conformal Cosmological Models, Phys. Rev. D 91 (2015) 067501 [arXiv:1407.8545] [INSPIRE].

[53] R. Jackiw and S.Y. Pi, New Setting for Spontaneous Gauge Symmetry Breaking?, Fundam. Theor. Phys. 183 (2016) 159 [arXiv:1511.00994] [INSPIRE].

[54] I. Antoniadis, E. Dudas and D.M. Ghilencea, Living with ghosts and their radiative corrections, Nucl. Phys. B 767 (2007) 29 [hep-th/0608094] [INSPIRE].

[55] R. Percacci, Scale Invariant Theories of Gravity and the Meaning of the Planck Mass, talk at Scale invariance in particle physics and cosmology, CERN, 28 January-01 February 2019 [https://indico.cern.ch/event/740038/contributions/3283831/].

[56] R. Percacci, Gravity from a Particle Physicists' perspective, PoS(ISFTG) 011 (2009) [arXiv: 0910.5167] [INSPIRE].

[57] R. Percacci, The Higgs phenomenon in quantum gravity, Nucl. Phys. B 353 (1991) 271 [arXiv:0712.3545] [INSPIRE]. 